\title{
Modeling and Simulation of Dynamic Reliability of Heating Pipe Network
}

\author{
Kuang Heng ${ }^{1, a}$ \\ ${ }^{1}$ CCTEG Chongqing Engineering Co., Ltd. Chongqing 400042, China \\ akuanghengcq@126.com
}

Keywords: heating network; repairable; numerical simulation; dynamic reliability evaluation

\begin{abstract}
Since 80s, the heating industry in China has been developing at an unprecedented speed, the rate of heating popularization, the scale of pipe network and the growth of the complexity are obvious. Due to the backward reliability design of the heating network in our country, the heating system is complex and diverse in the structure design. At the same time, because of the lack of equipment and the backward management level, the regulation of the system is not perfect. Facing the complex and diverse heating system in China, the traditional system cannot meet the requirements. The reliability evaluation of heating network is carried out by the method of property research. Therefore, the research on the dynamic reliability of heating system is not only the requirement of reliability evaluation of existing heating system in China, but also the urgent need of the safe operation of heating system.
\end{abstract}

\section{Introduction}

With the development of economy and society, people pay more and more attention to the living environment. The development of central heating not only alleviates the problems of energy shortage and serious environmental pollution, but also satisfies people in cold conditions. The requirement of high comfort in indoor environment ensures that the production and life of residents can proceed smoothly.

\section{Research on Reliability Evaluation Method of Domestic Heating Network}

The research on the reliability of heating network in our country began in the 1880s. Based on the research results of the former Soviet Union, some methods and measures to improve the reliability of the heating network put forward by optimizing the reliability evaluation index. Because of the probability index of no-accident operation of heating network put forward by the Moscow Institute of Architectural Engineering. At first, there are many hypotheses: assume that the system is irreparable, that the outdoor temperature changed during the heating period. In addition, that the outdoor temperature is the outdoor design temperature; when the failure occurs, the non-stop heating system can realize the limited heating, and the limited heating can guarantee the indoor temperature to be at the lowest allowable temperature. The design of heating system in China cannot meet the above hypothetical conditions, so Chinese scholars have modified and improved the probability of fault-free operation. Cai Qi lin put forward the concept of fault spectrum, and considered that the influence of fault occurred in different months, that is, different outdoor temperature, on the reliability of the system is different. According to the monthly fault frequency spectrum and the monthly outdoor average temperature, the fault spectrum coefficient is I Compared with the annual irreparable system of the former Soviet Union; the evaluation index has been greatly refined and improved. It considered that the system is irreparable in the month when the failure occurred, and is closer to the actual situation. It fixed the assumption of constant outdoor temperature during heating period. 


$$
R_{0(1)}=1-C_{f} \frac{\sum_{i=1}^{n} \Delta Q_{i} \lambda_{i}}{Q_{0} \sum_{i=1}^{n} \lambda_{i}}\left(1-e-\sum_{i=1}^{n} \lambda \beta\right)
$$

Among them, the frequency coefficient of the fault is as follows:

$$
C_{f}=\frac{\sum_{k-1}^{m}\left(t_{n}-t_{w k}\right) F_{k} h_{k}}{\sum_{k-1}^{m}\left(t_{n}-t_{w k}\right) h_{k}}
$$

In the form, $\mathrm{t}_{n}$ is the indoor design temperature, $\mathrm{t}_{\mathrm{wk}}$ is average outdoor temperature for the $\mathrm{k}$ month of the heating period, $h_{k}$ is heating hours for the month of $k, F_{k}$ is ratio of the number of failures to the total number of failures in the month of the $\mathrm{k}, \mathrm{M}$ is the number of months in which the heating period continues, The rest have the same formula as 1 . owing to $F_{k}<1$, so $C_{f}<1, R_{Z}(\mathrm{t})>R_{Z}(\mathrm{t})$.

Based on the theoretical study of the reliability of the former Soviet Union, he pointed out that the reliability evaluation index of the former Soviet Union had the following shortcomings: the assumption of the heating system as a non-repairable system was not objective. The criterion of the accident condition was not objective; the hypothesis of the stability of the fault flow in the completely heating period was not objective; the calculation worker was not objective. The hypothesis of the situation is not objective. After the study, we put forward the "monthly flow parameters", that is, the parameters of the failure flow of each month are constant, and the completely heating period divided into different months. It considered that the system is a month unrepeatable system, and the monthly average outdoor temperature used to replace the outdoor design temperature, and the reliability of the Poisson distribution formula obtained. Indicators

$$
R_{y}=1-\sum_{k-1}^{m} \frac{Q_{k o}}{Q_{y o}}\left(1-e^{-\varpi_{k i} z_{k}}\right) \sum_{k-1}^{n} \eta_{i} \frac{\varpi_{k i}}{\sum_{i=1}^{n} \varpi_{k i}}
$$

$Q_{\mathrm{ko}}$ for the design of the $\mathrm{k}$ month heat supply, $Q_{\mathrm{yo}}$ is heat supply for the whole heating period of the system, $\omega_{\mathrm{ki}}$ is an accident flow parameter, $Z_{K}$ is heating hours in the first month of the month, $\eta_{\mathrm{i}}=\Delta Q_{\mathrm{kj}} \div Q_{\mathrm{ko}}, \Delta Q_{\mathrm{ki}}$ is the $\mathrm{K}$ month i component failure caused by the insufficient amount of heating. Compared with the annual irreparable system of the former Soviet Union, the evaluation index has been greatly refined and improved. It considered that the system is irreparable in the month when the failure occurred, and is closer to the actual situation.

\section{Numerical Simulation of Failure Probability of Heating Network Components}

When the life distribution and maintenance time distribution of heating network elements known, the variation of failure probability of heating pipe network elements during the completely heating period can analyzed by numerical simulation method.

\subsection{Fault vector $g$}

The non-negative random variable $\mathrm{X}$ used to describe the life of the heating pipe network element, that is, the time from the beginning to the failure of the heating network element, and the corresponding distribution function is as follows $F(\mathrm{t})$ :

$$
F(\mathrm{t})=P(X \leq \mathrm{t})
$$


When the life distribution of heating network elements is exponential, the failure probability function is obtained of $F(\mathrm{t})$ :

$$
F(\mathrm{t})=1-\mathrm{e}^{-\lambda \mathrm{i}}
$$

Taking a heating cycle as the $T_{\max }$ life span of heating pipe network $\mathrm{t}$ simulation, Micro interval $\Delta t$ is the shortest maintenance time for heating pipe network elements. The simulation interval is $\mathrm{n}$, taking positive integer, Then the heating network elements in the case of irreparable in each micro-interval failure probability of $g_{i}(i=1,2, \ldots n)$ constitute a fault vector $g=\left\{g_{i}\right\}$ :

$$
\square \mathrm{g}_{i}=F[(i+1) \times \Delta t]-F[i \times \Delta t]
$$

\subsection{Maintenance vector $w$}

The time from the beginning of the failure to the completion of the repair of the component, that is, the time of fault diagnosis, maintenance preparation and maintenance implementation, is called the maintenance time of the component, it is a random variable denoted as Y. The maintenance time $\mathrm{Y}$ of the component distributed as $G_{(\mathrm{t})}$ the maintenance distribution.

$$
G_{(\mathrm{t})}=P(Y \leq t)
$$

When the maintenance time of heating pipe network elements distributed exponentially, the maintainability function of heating network elements is as $M_{(\mathrm{t})}$ follows:

$$
M_{(\mathrm{t})}=1-\mathrm{e}^{-\mu \mathrm{i}}
$$

When $M_{(\mathrm{t})}=0.99999$, Determination of maximum maintenance time for heating Pipe Network components is $\tau_{\max }$, The simulation interval is $\mathrm{m}$, taking positive integer, The maintenance probability of heating pipe network elements in each micro interval is $W_{\mathrm{i}}(\mathrm{i}=1,2, \ldots \mathrm{m})$ Component maintenance vector $w=\left\{w_{i}\right\}$ :

$$
w_{i}=M[(i+1) \times \Delta t]-M[i \times \Delta t]+\frac{0.99999}{m}
$$

\subsection{Repair sequence $r$}

A fault element at a certain time will maintained according to the probability law of the maintenance vector. The repair probability of components in each interval during operation constitutes a repair sequence is $r$. When $t=0, r_{0}=1$, indicates that all the components are in normal condition, and $r_{1}, r_{2}, \ldots r_{n}=0$, The amount of repair and the frequency of repair are expressed $r_{i}$ in terms of the quantity and frequency of repair. The repair sequence calculated as follows:

When the simulation interval is $\mathrm{i} \leq \mathrm{m}$

$$
\mathrm{r}_{\mathrm{i}}=\sum_{\mathrm{j}-1}^{\mathrm{i}} g_{i}-j+1^{w_{j}}
$$

When the simulation interval is $\mathrm{i}>m$

$$
\mathrm{r}_{i}=\sum_{j-1}^{\mathrm{m}} r_{i}-j+1^{w_{j}}
$$

\subsection{Fault frequency sequence $g^{\mathrm{w}}$}

The fault frequency sequence ${ }^{w}{ }^{w}$ refers to the maintainable condition, The probability of failure 
of the component in the micro interval. Therefore, the fault frequency sequence describes the operation process of the component under the condition of maintenance. The calculation is as follows:

When the simulation interval is $\mathrm{i} \leq \mathrm{n}$

$$
\mathrm{g}_{i}{ }^{w}=\sum_{j-1}^{i} r_{i}-{ }_{j} g_{j}
$$

When the simulation interval is $\mathrm{i}>n$

$$
\mathrm{g}_{i}{ }^{w}=\sum_{j-1}^{n} r_{i}-{ }_{j} g_{j}
$$

\section{No Accident Work Probability Index of Repairable Heating Network $R_{\mathrm{dy}}$}

Since the no-accident nature of the heating network can reflect the functional reliability of the heating network, one of the most important numerical indexes of the reliability of the heating system is the no-accident working probability. By analyzing the insufficiency of traditional reliability evaluation index, considering the dynamic factors that affect the reliability of heating system, such as outdoor air temperature, heating duration and so on, this paper improves the probability index of non-accident operation of irreparable pipe network. The reliability evaluation index of the dynamic function of the heating system put forward, which can used to repair the probability index of the heating network without accident $R_{\mathrm{dy}}$.

Theoretical heat $Q_{\mathrm{k}}$ supply of heating system $\mathrm{t}_{w k}$ with outdoor temperature. That is the heating system is in a no-accident state of heat supply; $Q_{\mathrm{ki}}$ is outdoor temperature ,The actual heat $\mathrm{t}_{w k}$ supply when the $\mathrm{i}$ accident element causes the accident of the heating system; $\Delta Q_{\mathrm{ki}}$ is outdoor temperature, The insufficient $t_{w k}$ amount of heat supply in the event of the system accident caused by the i accident element, $N_{\mathrm{k}}$ is outdoor temperature , $\mathrm{t}_{w k}$ is the heating duration, Then:

$$
\Delta Q_{\mathrm{ki}}=Q_{k o}-Q_{k i}
$$

The expectation of actual heat supply for outdoor heating system is as follows:

$$
E\left(Q_{\mathrm{k}}\right)=Q_{k o} P_{k o}\left(N_{k}\right)+\sum_{i=1}^{n} Q_{k i} P_{k i}\left(N_{k}\right)=Q_{k o}-\sum_{i=1}^{m} \Delta Q_{k i} P_{k i}\left(N_{k}\right)
$$

Among them, $\mathrm{m}$ is the number of accident elements in the heating network; this is the instantaneous state probability of the solved fault element. The ideal state of heat supply for a heating network during the whole heating period is $Q_{\mathrm{yo}}$ as follows. The mathematical expectation of actual heat supply is $E\left(Q_{\mathrm{y}}\right)$ :

$$
Q_{\mathrm{yo}}=\sum_{k-1}^{j} Q_{k o}
$$

\section{Conclusion}

In this paper, the fault state and accident state of repairable heating network are distinguished at first and different from the traditional reliability research, the criterion of fault determination put forward, and the normal state of repairable heating network described by using the reliability model of stochastic Petri net. Through analysis, it concluded that the availability of pipeline network is higher than that of non-repairable pipe network when maintenance is considered, and the availability of pipe network increases with the increase of outdoor temperature. With the increase of 
heating capacity, the probability index of fault-free operation has the same law, and the average fault time and the average accident time of pipe network are shortened with the increase of outdoor temperature, which indicates that the higher the outdoor temperature, the shorter the time the pipe network is in trouble or accident.

\section{References}

[1] Wang Xiao x. Study on failure condition and Reliability of Ring Hot Water Pipe Network with multiple Heat sources [D]. Harbin: Ph. D. thesis, Harbin University of Technology, 2004

[2] District Heating in Denmark[M]. Danish Energy Agency. 1993: 1-4.

[3] Schmitz, Karin. District heat supply in Germany in the year 2001[J]. Euroheat and Power. 2003, 32(1-2): 28-35.

[4] Wang S j, Li D y, Yan Q. French urban central heating introduction [J]. regional heating. 2002, (3): 29-31.

[5] D J-y, F J-b, J Zhen, et al. CJJ 34-2010 CJJ. China Construction Industry Press, 2010.

[6] Z Ping H, Wang Wei, Wang P. New Progress in the study of the Reliability of Heat Supply Networks in Russia: An Analysis of the New Code of Russia 41-02-2003 [J]. District heating, 2012 / 2: 7-13.

[7] P Wang, P h Z. Simulation of Distributed Pump Systems under Failure Conditions[C]. Proceedings of Second International Conference on Modeling and Simulation Vol. 3, Manchester, England, UK, 2009: 118 123.

[8] Qin X z, Jiang Yi. Analysis of the accessibility of ring heating network based on genetic algorithm [J]. Journal of Q h University: natural Science Edition, 1999, 39D 6N: 90-94.

[9] Wang Wei. Study on reliability of heat supply system based on graph theory [D]. Harbin: Doctoral Dissertation of Harbin Institute of Technology, 2010. 